\title{
MAPPING GALOIS EXTENSIONS INTO DIVISION ALGEBRAS
}

\author{
NIKOLAUS VONESSEN
}

(Communicated by Maurice Auslander)

\begin{abstract}
Let $A$ be a ring with a finite group of automorphisms $G$, and let $f_{1}$ and $f_{2}$ be homomorphisms from $A$ into some division algebra $D$ such that $f_{1}$ and $f_{2}$ agree on the fixed ring $A^{G}$. Assuming certain additional assumptions, it is shown that $f_{1}$ and $f_{2}$ differ only by an automorphism in $G$ and an inner automorphism of $D$.
\end{abstract}

\section{INTRODUCTION}

Let $A$ be a commutative ring with a finite group $G$ of automorphisms, and let $f_{1}$ and $f_{2}$ be maps from $A$ into some field $D$ such that $\left.f_{1}\right|_{A^{G}}=\left.f_{2}\right|_{A^{G}}$. Then there is some $\sigma \in G$ such that $f_{2}=f_{1} \circ \sigma$ [B, Chapter V, $\S 2$, no. 2, Corollary to Theorem 2]. We generalize this result to the setting of noncommutative rings and division algebras, proving - under certain additional assumptionsthe existence of some $\sigma \in G$ and some inner automorphism $\psi$ of $D$ such that $f_{2}=\psi \circ f_{1} \circ \sigma$ (Theorems 1 and 2 below).

We begin with a simple but instructive example due to Montgomery, which shows why inner automorphisms are needed in the noncommutative case. Let $A=\mathrm{C}$ denote the complex numbers, and let $G$ be the group of automorphisms of $A$ generated by complex conjugation. Then $A^{G}=\mathbf{R}$. Let $f_{1}$ be the natural inclusion of $A$ into the quaternions $D=\mathbf{H}$, and let $f_{2}: A \rightarrow D$ be the R-linear map defined by $f_{2}(i)=j$. Then $\left.f_{1}\right|_{A^{G}}=\left.f_{2}\right|_{A^{G}}$, but $f_{1}(A) \neq f_{2}(A)$. Hence for all $\sigma \in G, f_{2} \neq f_{1} \circ \sigma$. Montgomery noted, however, that the Skolem-Noether theorem implies the existence of some inner automorphism $\psi$ of $D$ such that $f_{2}=\psi \circ f_{1}$. (E.g., let $\psi$ be conjugation by $i+j$.) This led her to conjecture that in generalizing the commutative result, one should aim to prove $f_{2}=\psi \circ f_{1} \circ \sigma$. In this direction, we obtained the following two results:

Theorem 1. Let $A$ be a ring with a finite group of automorphisms $G$ such that $|G|^{-1} \in A$. Let $D$ be a division algebra, and let $f_{1}$ and $f_{2}$ be homomorphisms

Received by the editors April 3, 1992.

1991 Mathematics Subject Classification. Primary 16W20; Secondary 16K99, 12E15, 13 B05.

Partially supported by NSF grant DMS 8901491. The author gratefully acknowledges the hospitality of the Instituto de Matemática Pura e Aplicada, Rio de Janeiro, and of the Universidade Federal do Rio de Janeiro while the final version of this paper was written. 
from $A$ into $D$ such that $\left.f_{1}\right|_{A^{G}}=\left.f_{2}\right|_{A^{G}}$. Suppose that $f_{1}(A)$ is a ring satisfying a polynomial identity. Then there is some $\sigma \in G$ and some inner automorphism $\psi$ of $D$ such that $f_{2}=\psi \circ f_{1} \circ \sigma$.

A few remarks are in order. First of all, there are two interesting cases where the hypothesis that $f_{1}(A)$ be a polynomial identity ring is trivially satisfied: If either $A$ itself is a PI-ring, in particular, if $A$ is actually commutative, or if $D$ is finite dimensional over its center. I do not know if the PI-hypothesis is necessary. It is certainly needed in the proof in an essential way; cf. Remark 5 below.

Secondly, note that the map $\psi$ in the theorem, when restricted to $f_{1}\left(A^{G}\right)$, is the identity. Given any map $f: A \rightarrow D$, call $f$ equivalent to $f_{1}$ if $f=$ $\psi \circ f_{1} \circ \sigma$, where $\sigma \in G$ and where $\psi$ is any inner automorphism of $D$ with $\left.\psi\right|_{f_{1}\left(A^{G}\right)}=\mathrm{id}_{f_{1}\left(A^{G}\right)}$. Trivially, equivalent maps agree on the fixed ring $A^{G}$. The theorem says that all maps which agree on $A^{G}$ are equivalent! This is quite surprising. For example, another way to construct maps which agree on $A^{G}$ is as follows: Given any automorphism $\tau$ of $A$ which induces the identity on $A^{G}$, the maps $f_{1}$ and $f_{1} \circ \tau$ agree on $A^{G}$. So by the theorem, $f_{1}$ and $f_{1} \circ \tau$ are equivalent, i.e., $f_{1} \circ \tau=\psi \circ f_{1} \circ \sigma$, although $\operatorname{Aut}_{A^{G}}(A)$ is in general much bigger than $G$. (Of course, this situation occurs already in the commutative setting, but only if $f_{1}$ is not injective. In the noncommutative setting, it appears even if $A$ is a division algebra. And we will see that-given a theorem of Montgomery-it is fairly easy to reduce the proof of Theorem 1 to the latter case; see $\S 3$.)

Thirdly, in general one cannot drop the assumption that $|G|^{-1} \in A$, even if $A$ is an affine prime Noetherian PI-ring finite over its center and if $D$ is a (commutative) field (Example 7). However, we have the following positive result:

Theorem 2. Let $A$ be a ring with a finite group of automorphisms $G$. Let $D$ be a division algebra, and let $f_{1}$ and $f_{2}$ be homomorphisms from $A$ into $D$ such that $\left.f_{1}\right|_{A^{G}}=\left.f_{2}\right|_{A^{G}}$. Suppose that either

(a) $f_{1}$ is injective and $A$ satisfies a polynomial identity, or

(b) $A$ is commutative and $D$ is finite over its center.

Then there is some $\sigma \in G$ and some inner automorphism $\psi$ of $D$ such that $f_{2}=\psi \circ f_{1} \circ \sigma$.

In generalizing the commutative result, one could also try to replace the field $D$ by a finite-dimensional central simple algebra. We will see later in Example 8 that this approach does not work, even if $A$ is commutative.

I should mention a related result on equivalence of maps by $\mathrm{F}$. Pop and $\mathrm{H}$. Pop [PP]. They proved among other things the following: Let $A$ be a semisimple algebra over a field $k$, and let $D$ be a separable $k$-algebra. Then up to composition by inner automorphisms of $D$, there are only finitely many algebra homomorphisms from $A$ to $D$.

This paper is organized as follows. Section 2, the heart of this paper, deals with the special case that $A$ is a division algebra. Section 3 contains the proofs of the main theorems, and $\S 4$ consists of two examples.

Finally, I would like to thank Susan Montgomery for bringing this problem to my attention. 


\section{The DIVISION ALGEBRA CASE}

The following proposition is the basic technical result we will need. It is the special case that $A=S$ is a division algebra. Note that here we make no assumptions on the order of $G$.

Proposition 3. Let $S$ be a division algebra finite over its center $K$. Let $G$ be a finite group of automorphisms of $S$. Let $f_{1}$ and $f_{2}$ be embeddings of $S$ into a division algebra $D$ such that $\left.f_{1}\right|_{K^{G}}=\left.f_{2}\right|_{K^{G}}$. Then there is some $\sigma \in G$ and some inner automorphism $\psi$ of $D$ such that $f_{2}=\psi \circ f_{1} \circ \sigma$.

Let $k$ be some subfield of $K^{G}$ such that $K^{G}$ is a finite purely inseparable extension of $k$. The proof will show that it is actually sufficient to require only that $\left.f_{1}\right|_{k}=\left.f_{2}\right|_{k}$. Moreover, if $D$ is finite over its center, $K^{G}$ may be an infinite purely inseparable extension of $k$. We will prove the proposition in this slightly greater generality. This will be important in the proof of Theorem 2(b).

Proof. We first show that we may assume that $S \subseteq D$, and that $f_{1}$ is the natural embedding. Namely, if the proposition is true in the latter case, then $f_{2} \circ f_{1}^{-1}=\psi \circ \sigma^{\prime}$ for some $\sigma^{\prime} \in\left(f_{1} \circ G \circ f_{1}^{-1}\right)$ and some $\psi \in \operatorname{Inn}(D)$. Say $\sigma^{\prime}=f_{1} \circ \sigma \circ f_{1}^{-1}$ for $\sigma \in G$. Then $f_{2}=\psi \circ\left(\sigma^{\prime} \circ f_{1}\right)=\psi \circ f_{1} \circ \sigma$, as desired. So from now on we will assume that $S \subseteq D$, and that $f_{1}$ is the natural embedding. To simplify notation, set $f=f_{2}, T=f(S)$, and $L=f(K)$. Then $\left.f\right|_{k}=\mathrm{id}_{k}$, and $L$ is the center of $T$. We have to find $\sigma \in G$ and $\psi \in \operatorname{Inn}(D)$ such that $f=\psi \circ \sigma$. The following figure illustrates the situation:

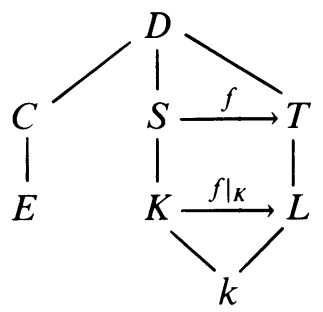

Here $C$ denotes the center of $D$, and $E$ always denotes a subfield of $C$.

In the sequel, we will work with various division subalgebras of $D$ generated by some subfield $E$ of $C$ and either $S$ or $T$. For example, $E S$ denotes the division algebra generated by $E$ and $S$ inside $D$. If $E K$ denotes the compositum of the fields $E$ and $K$ inside $D$, the $E K \otimes_{K} S$ is a simple ring with center $E K$. The natural map from $E K \otimes_{K} S$ into $E S$ is thus injective; hence $E K \otimes_{K} S$ is a domain. Since $S$ is finite over $K, E K \otimes_{K} S$ is finite over the field $E K$. Thus it is a division algebra and therefore isomorphic to $E S$. To summarize, $E S \approx E K \otimes_{K} S$ is a division algebra which is finite dimensional over its center $E K$. (See Remark 5 below for a discussion of what would happen if $S$ were not finite over $K$.)

Let $D^{\prime} \subseteq D$ be the division algebra generated by $C S$ and $C T$. Then $C k$ is central in $D^{\prime}$. (This follows immediately from the construction of $D^{\prime}$ : Let $R_{1}$ be the subring of $D$ generated by $C S$ and $C T$, and for any integer $i>1$, let $R_{i}$ be the subring of $D$ generated by $R_{i-1}$ and the inverses of all nonzero elements in $R_{i-1}$. Then $D^{\prime}=\bigcup_{i} R_{i}$ is a division algebra, and $C k$ is central in $D^{\prime}$ since it is central in all $R_{i}$.) Since every inner automorphism of $D^{\prime}$ extends to $D$, we may therefore replace $D$ by $D^{\prime}$ and thus assume that $k$ is contained in the center $C$ of $D$. 
We will see in Lemma 4 below that for some $\sigma \in G, f \circ \sigma$ extends to an isomorphism $g: C S \rightarrow C T$ such that $\left.g\right|_{C}=\mathrm{id}_{C}$. If $K^{G}$ is a finite extension of $k$, then $C K$ is finite over $C k=C$. Since $S$ is finite over $K, C S \approx C K \otimes_{K} S$ is finite over $C K$. Thus $C S$ is finite over $C$, and so is $C T=g(C S)$. If $K^{G}$ is not finite over $k, D$ is finite over $C$ by assumption. So in any event, now both $C S$ and $C T$ are finite dimensional over $C$. It follows by the Skolem-Noether theorem that $g$ is the restriction of some inner automorphism $\psi$ of $D$. Since $\left.\psi\right|_{S}=\left.g\right|_{S}=f \circ \sigma$, we conclude that $f=\psi \circ \sigma^{-1}$, proving the proposition modulo the following lemma.

Lemma 4. There is a $\sigma \in G$ such that $f \circ \sigma$ extends to an isomorphism $g: C S \rightarrow$ $C T$ such that $\left.g\right|_{C}=\mathrm{id}_{C}$.

Proof. Let $\mathscr{M}$ be the set of all tuples $(E, h)$ such that $E$ is a subfield of $C$ containing $k$ and $h$ is an isomorphism $E S \rightarrow E T$ extending $f$ and inducing the identity on $E$. Note that $\mathscr{M} \neq \varnothing$ since $(k, f) \in \mathscr{M}$. We introduce a partial ordering on $\mathscr{M}$ by setting $(E, h) \leq\left(E^{\prime}, h^{\prime}\right)$ iff $E \subseteq E^{\prime}$ and $\left.h^{\prime}\right|_{E S}=h$. We next show that $\mathscr{M}$ is inductively ordered. Let $\left\{\left(E_{i}, h_{i}\right)\right\}$ be a chain in $\mathscr{M}$. Then $E=\bigcup_{i} E_{i}$ is a subfield of $C$. Since $E S \approx E K \otimes_{K} S$, every element $\alpha$ of $E S$ involves only a finite number of elements of $E$. Thus $\alpha \in E_{i} S$ for some $i$. It follows easily that the $h_{i}$ extend to an isomorphism $h: E S \rightarrow E T$ inducing the identity on $E$. Hence $\mathscr{M}$ is inductively ordered.

Thus Zorn's lemma implies the existence of a maximal element $\left(E_{1}, g_{1}\right)$. Assume that there is a subfield $E_{2}$ of $C$ properly containing $E_{1}$ and an isomorphism $g_{2}: E_{2} S \rightarrow E_{2} T$ inducing the identity on $E_{2}$ and extending $f \circ \sigma_{2}$ for some $\sigma_{2} \in G$. Choose $\left(E_{2}, g_{2}\right)$ maximal with respect to these properties, and continue. Since $G$ is finite, we eventually obtain a subfield $E$ of $C$ maximal with respect to the following property $(*)$ : There is an isomorphism $g: E S \rightarrow E T$ inducing the identity on $E$ and extending $f \circ \sigma$ for some $\sigma \in G$. Since we do not mind replacing $f$ by $f \circ \sigma$, we can as well assume that $g$ extends $f$.

Let $\alpha$ be an element of $C$. We will show that $\alpha \in E$. Let $E S[x]$ and $E T[x]$ be the polynomial rings in one central indeterminate over the division algebras $E S$ and $E T$. Consider the homomorphisms $\Phi: E S[x] \rightarrow E S[\alpha]$ and $\Psi: E T[x] \rightarrow E T[\alpha]$ sending $x$ to $\alpha$. The kernel of $\Phi$ is generated by a polynomial $q(x)$ with central coefficients [MR, 9.6.3]. That is, $q(x) \in E K[x]$. It follows that $q(x)=0$ iff $\operatorname{Ker} \Phi=0$ iff $\alpha$ is transcendent over $E K$ iff $\alpha$ is transcendent over $E$ iff $\alpha$ is transcendent over $E L$ iff $\operatorname{Ker} \Psi=0$. Here we used that both $E K$ and $E L$ are algebraic over $E$. Assume that $\operatorname{Ker} \Phi=0$. Then it follows by the above remarks that $\alpha$ is a central indeterminate over both $E S$ and $E T$. Set $E^{\prime}=E(\alpha)$. Then $g$ extends to an isomorphism $g^{\prime}: E^{\prime} S \rightarrow$ $E^{\prime} T$ by defining $g^{\prime}(\alpha)=\alpha$. Clearly $g^{\prime}$ extends $f$, and $\left.g^{\prime}\right|_{E^{\prime}}=\mathrm{id}_{E^{\prime}}$. This is a contradiction to the maximality of $E$. Hence $\operatorname{Ker} \Phi \neq 0$, and $\alpha$ is algebraic over $E K$ with irreducible polynomial $q(x)$.

Consider the polynomial $p(x)=\prod_{\sigma} \sigma(q(x))$, where $\sigma$ runs over all elements in $\operatorname{Gal}(E K / E)$. Since $K$ is normal over $k, E K$ is normal over $E$. If $K$ is separable over $k$, then also $E K$ is separable over $E$, and $p(x)$ belongs to the polynomial ring $E[x]$. Otherwise, some power of $p(x)$ belongs to $E[x]$; we denote that power again by $p(x)$. Since $g(p(x))=p(x), g(p(\alpha))=0$. It follows that for some $\sigma \in \mathrm{Gal}(E K / E), g(\sigma(q(x)))$ is the irreducible polynomial of $\alpha$ over $E L$. That is, the kernel of $\Psi$ is generated by $g(\sigma(q(x)))$. 
We claim that $\sigma$, which is an automorphism of $E K$, extends to an automorphism of $E S$ stabilizing $S$. Since $K$ is normal over $k$, and since $k \subseteq E$ is pointwise fixed under $\sigma,\left.\sigma\right|_{K}$ is an automorphism of $K / k$. Since the group $G$ of automorphisms of $S$ maps via restriction onto $\operatorname{Gal}\left(K / K^{G}\right)=\operatorname{Gal}(K / k)$, there is some $\tau \in G$ such that $\left.\tau\right|_{K}=\left.\sigma\right|_{K}$. Hence $\sigma \otimes \tau$ induces an automorphism of $E S \approx E K \otimes_{K} S$ extending both $\sigma$ and $\tau$. We denote this automorphism again by $\sigma$.

Let $E^{\prime}=E(\alpha)$. As seen above, the irreducible polynomials of $\alpha$ over $E K$ and $E L$ are $q(x)$ and $g(\sigma(q(x)))$, respectively. Hence $g \circ \sigma: E S \rightarrow E T$ extends to an isomorphism $g^{\prime}: E^{\prime} S \rightarrow E^{\prime} T$ by setting $g^{\prime}(\alpha)=\alpha$. Clearly $\left.g^{\prime}\right|_{E^{\prime}}=\mathrm{id}_{E^{\prime}}$ and $g^{\prime}$ extends $f \circ \tau$. This is a contradiction to the maximality of $E$, unless $\alpha \in E$. It follows that $E=C$, completing the proof of the lemma, and thus the proof of Proposition 3.

As an immediate corollary, we obtain Theorem 2(a):

Proof of Theorem 2(a). Since $A$ is a PI-domain, its division ring of fractions $\mathrm{Q}(A)$ can be obtained by a central localization. One concludes easily that $A^{G}$ has also a division ring of fractions $\mathrm{Q}\left(A^{G}\right)$, and that $\mathrm{Q}\left(A^{G}\right)=\mathrm{Q}(A)^{G}$. Now the result follows immediately from Proposition 3.

Remark 5. Where in the proof of Proposition 3 did we use the fact that $S$ is finite over its center $K$ ? Certainly via the Skolem-Noether theorem: There we needed it to show that $C S$ is finite over $C$. And this is in fact the only place where we needed this assumption: Assume that $S$ is not necessarily finite over its center $K$. If again $E$ is an arbitrary subfield of $C$, and if $R$ denotes the image of $E K \otimes_{K} S$ in $E S$, then $R$ is now not necessarily equal to $E S$. But one can check that $R$ is an Ore domain, so that $S \approx \mathrm{Q}\left(E K \otimes_{K} S\right)$. Moreover, the center of $\mathrm{Q}\left(E K \otimes_{K} S\right.$ ) is still $E K$ (if $c$ belongs to the center of $\mathrm{Q}(R)$, then $\{r \in R \mid c r \in R\}$ is a nonzero two-sided ideal of $R$ and thus all of $R$; hence $c \in R$, so that the center of $\mathrm{Q}(R)$ is isomorphic to $E K)$. Using these facts, the proof of Lemma 4 goes through. In order to attempt a generalization of Proposition 3 to the case of an arbitrary division algebra $S$, we reduced thus to the case that $f$ extends to a map $C S \rightarrow C T$ with $\left.f\right|_{C}=\mathrm{id}_{C}$. It is not clear if this is of any help. I should remark that the PI-assumption in Theorem 1 is also used in the proof in the next section: There we need it to show that a certain prime homomorphic image $A / P$ of $A$ is a Goldie ring. Of course, one could assure this by assuming, e.g., that $A$ is Noetherian.

\section{THE PROOFS OF THE MAIN THEOREMS}

These proofs consist now of reduction steps to the case that $A$ is a division algebra. First we need the following lemma which incorporates a general reduction technique which is sometimes useful when studying actions of finite groups on rings. It is most likely known, but included for lack of a reference.

Lemma 6. Let $A$ be a ring with a finite group of automorphisms $G$ such that $|G|^{-1} \in A$. Let $P$ be a prime ideal of $A$ such that $A / P$ is a right Goldie ring. Set $H=\operatorname{Stab}_{G}(P)$ and $I=\bigcap_{\sigma \in G} \sigma(P)$. Then

$$
\mathrm{Q}\left(A^{G} / P \cap A^{G}\right) \approx \mathrm{Q}(A / I)^{G} \approx \mathrm{Q}(A / P)^{H},
$$

where the isomorphisms are induced by the quotient map $A \rightarrow A / P$. 
Note that here $G$ acts on $A / I$ but not necessarily on $A / P$. This lemma is useful in that it relates-on the quotient ring level-the actions of $G$ on $A$ and $A / I$ with the action of $H$ on $A / P$, allowing in certain situations the reduction from arbitrary rings to prime rings. We shall use it in the proof of Theorem 1 .

One should note that the lemma does not hold "before" localization. Let me be more precise. Recall that if a group $G$ acts on a semiprime right Goldie ring $R$ such that $|G|^{-1} \in R$, theri also $R^{G}$ is a semiprime right Goldie ring, and $\mathrm{Q}\left(R^{G}\right)=\mathrm{Q}(R)^{G}$ [MR, 10.5.19]. We will use these facts frequently. Thus the lemma says that the quotient rings of $A^{G} / P \cap A^{G}$ and $(A / P)^{H}$ are isomorphic. However, it is in general not true that $A^{G} / P \cap A^{G}$ and $(A / P)^{H}$ are isomorphic, even if $A$ is commutative; see [B, Chapter V, $\S 2$, no. 2, Exercise 10].

Proof. Note that $P \cap A^{G}=I \cap A^{G}$, so that $A^{G} / P \cap A^{G}=A^{G} / I \cap A^{G} \approx(A / I)^{G}$. Thus $\mathrm{Q}\left(A^{G} / P \cap A^{G}\right) \approx \mathrm{Q}(A / I)^{G}$, and this isomorphism is induced by the quotient map $A / I \rightarrow A / P$. To prove the second isomorphism, we may as well replace $A$ by $A / I$ and thus assume that $I=0$. It is easy to see that $A$ is now a semiprime right Goldie ring. (Use the embedding $A \hookrightarrow \bigoplus_{\sigma \in G} A / \sigma(P)$, and the fact that the latter ring is right Goldie.) Note that $G$ permutes the minimal prime ideals of $A$ transitively. Denote by $B$ the total ring of fractions of $A$. Then $B$ is a direct sum of simple Artinian rings, say $B_{1}, \ldots, B_{n}$. The action of $G$ on $A$ extends to $B$, and $G$ permutes the $B_{i}$ transitively. Say $Q=B_{2} \oplus \cdots \oplus B_{n}$ is the prime ideal of $B$ which is the localization of $P$. Then $B_{1} \approx B / Q \approx \mathrm{Q}(A / P)$. Note that $H=\operatorname{Stab}_{G}(P)=\operatorname{Stab}_{G}\left(B_{1}\right)$.

The projection from $B$ onto $B_{1}$ induces a homomorphism from $B^{G}$ into $B_{1}^{H}$. Since $G$ permutes the $B_{i}$ transitively, this map is one-to-one. It is actually also onto, as one can see as follows: Let $\sigma_{i}$ be right coset representatives of $H$ in $G$ such that $\sigma_{i}\left(B_{1}\right)=B_{i}$. Let $b \in B_{1}^{H}$, and set $x=\sum_{i} \sigma_{i}(b)$. Then given $\tau \in G$, there is a permutation $\pi$ and elements $h_{i} \in H$ such that $\tau \sigma_{i}=\sigma_{\pi(i)} h_{i}$. Thus $\tau(x)=\sum_{i} \sigma_{\pi(i)}\left(h_{i}(b)\right)=x$. Hence $x \in B^{G}$, and the image of $x$ in $B_{1}^{H}$ is $b$. Thus $B^{G} \approx B_{1}^{H}$, i.e., $\mathrm{Q}(A)^{G} \approx \mathrm{Q}(A / P)^{H}$, and this isomorphism is induced by the quotient map $A \rightarrow A / P$.

Proof of Theorem 1. Denote by $P_{i}$ the kernel of $f_{i}$. Then $P_{1} \cap A^{G}=P_{2} \cap A^{G}$. It follows by a theorem of Montgomery $\left[\mathrm{M}_{1}\right]$ (see also $\left[\mathrm{M}_{2}\right]$ ) that there is some $\sigma \in G$ such that $\sigma\left(P_{2}\right)=P_{1}$. Replacing $f_{1}$ by $f_{1} \circ \sigma$, we may therefore assume that $f_{1}$ and $f_{2}$ have the same kernel $P$. Moreover, $A / P$ is now by assumption a PI-ring and thus a Goldie ring. So the hypotheses of Lemma 6 are satisfied. It follows that the maps $f_{i}$ induce maps $\bar{f}_{i}: \mathrm{Q}(A / P) \rightarrow D$ which agree on $\mathrm{Q}(A / P)^{H} \approx \mathrm{Q}\left(A^{G} / P \cap A^{G}\right)$. Hence by Proposition 3, there is some $\sigma \in H$ and some inner automorphism $\psi$ of $D$ such that $\bar{f}_{2}=\psi \circ \bar{f}_{1} \circ \sigma_{\mathrm{Q}(A / P)}$. (If $\sigma$ acts on a ring $R$, we denote here the corresponding automorphism by $\sigma_{R}$.) We conclude that $f_{2}=\psi \circ f_{1} \circ \sigma_{A}$, as was to be shown.

Proof of Theorem 2(b). Using Proposition 3 instead of some elementary facts about commutative fields, this result follows exactly like [B, Chapter V, $\S 2$, no. 2, Corollary to Theorem 2]. The proof is only included for completeness.

Denote by $P_{i}$ the kernel of $f_{i}$. Then $P_{1} \cap A^{G}=P_{2} \cap A^{G}$. Thus there is some $\sigma \in G$ such that $\sigma\left(P_{2}\right)=P_{1}$. Replacing $f_{1}$ by $f_{1} \circ \sigma$, we may therefore assume that $f_{1}$ and $f_{2}$ have the same kernel $P$. Denote by $K$ and $k$ the fields of fractions of $A / P$ and $A^{G} / P \cap A^{G}$, respectively. Then $f_{1}$ and $f_{2}$ induce 
embeddings $\bar{f}_{1}$ and $\bar{f}_{2}$ of $K$ into $D$ such that $\left.\bar{f}_{1}\right|_{k}=\left.\bar{f}_{2}\right|_{k}$. By [B, loc. cit., Theorem 2], $K / k$ is normal with finite Galois group $H$. By Proposition 3 applied to $S=K$, there is some $\bar{\sigma} \in H$ and some inner automorphism $\psi$ of $D$ such that $\bar{f}_{2}=\psi \circ \bar{f}_{1} \circ \bar{\sigma}$. Every element $\alpha \in \operatorname{Stab}_{G}(P)$ defines an automorphism $\bar{\alpha}$ of $A / P$ which extends to $K$ and leaves $k$ pointwise fixed. Again by the already cited theorem in [B], the map $\operatorname{Stab}_{G}(P) \rightarrow H$ sending $\alpha$ to $\bar{\alpha}$ is onto. Hence $\bar{\sigma}$ comes from some $\sigma \in \operatorname{Stab}_{G}(P)$. It follows that $f_{2}=\psi \circ f_{1} \circ \sigma$.

\section{EXAMPLeS}

First we show that in general it is impossible in the noncommutative case to drop the assumption that $|G|^{-1} \in A$, even if $A$ is an affine prime Noetherian PI-algebra finite over its center, and $D$ is a (commutative) field. It is fairly simple to find such examples where $f_{1}$ and $f_{2}$ have kernels which are not conjugate under the group action. Essentially, examples of this type are based on the fact that Montgomery's theorem does not hold if $|G|$ is not invertible in $A$. In the following somewhat more complicated example, $f_{1}$ and $f_{2}$ have the same kernel and agree on $A^{G}$, but are anyhow not equivalent.

Example 7. Let $R=k[x, t]$ be a polynomial ring in two variables over a field of prime characteristic $p \neq 2$. Let

$$
A=\left(\begin{array}{cc}
R & R \\
t R & k\left[x^{2}\right]+t R
\end{array}\right) .
$$

Then $A$ is an affine prime Noetherian PI-algebra finite over its center $\left(k\left[x^{2}\right]+t R\right) \cdot I_{2}$. Let $G$ be the group of automorphisms of $A$ generated by conjugation by $\left(\begin{array}{ll}1 & 1 \\ 0 & 1\end{array}\right)$. Then $|G|=p$, and $A^{G}=\left(k\left[x^{2}\right]+t R\right) \cdot I_{2}+R \cdot e_{12}$. Let $P$ be the prime ideal

$$
P=\left(\begin{array}{cc}
t R & R \\
t R & k\left[x^{2}\right]+t R
\end{array}\right) .
$$

Then $A / P=k[x]$ and $A^{G} / P \cap A^{G}=k\left[x^{2}\right]$. (Note that $A^{G} / P \cap A^{G} \neq(A / P)^{G}=$ $k[x]$; if $|G|$ were invertible in $A$, equality would hold.) Let $D=k(x)$, and let $f_{1}$ be the composition $A \rightarrow A / P \hookrightarrow D$. Let $f_{2}$ be the composition of $f_{1}$ with the automorphism of $D$ which sends $x$ to $-x$. Then $f_{1}$ and $f_{2}$ agree on $A^{G}$. But $f_{1}$ and $f_{2}$ are not equivalent: The field $D$ admits only the trivial inner automorphism $\psi=\mathrm{id}$. And if $\sigma \in G$, then $f_{1}=f_{1} \circ \sigma$ since $\sigma$ is inner and $f_{1}(A)=A / P$ is commutative. Thus $\psi \circ f_{1} \circ \sigma=f_{1} \neq f_{2}$.

Our last example shows that in generalizing the commutative result, one cannot replace the field $D$ by a finite-dimensional central simple algebra, even if $A$ is commutative.

Example 8. Let $A=\mathrm{C}$ be the complex numbers, and let $G$ be the group of automorphisms of $A$ given by complex conjugation. Let $D$ be the $(2 \times 2)$ matrices over $\mathbf{C}$. Let $f_{1}$ be the natural embedding of $A$ as the center of $D$, and let $f_{2}$ be the map sending $a \in \mathbf{C}$ to the matrix $\left(\begin{array}{l}a \\ 0\end{array} \frac{0}{a}\right)$. Then $f_{2}(A)$ is not central in $D$, so that for all automorphisms $\psi$ of $D, f_{2}(A) \neq \psi\left(f_{1}(A)\right)$. 


\section{REFERENCES}

[B] N. Bourbaki, Elements of mathematics, commutative algebra, Chapters 1-7, Springer, Berlin, 1989. Originally published as: N. Bourbaki, Éléments de mathématique, algèbre commutative, Chapitres 5 \& 6, Hermann, Paris, 1964.

[MR] J. C. McConnell and J. C. Robson, Noncommutative Noetherian rings, Wiley-Interscience, New York, 1989.

[M $\mathrm{M}_{1}$ S. Montgomery, Prime ideals in fixed rings, Comm. Algebra 9 (1981), 423-449.

$\left[\mathrm{M}_{2}\right] \quad-$, Prime ideals and group actions in noncommutative algebras, Contemp. Math., vol. 88, Amer. Math. Soc., Providence, RI, 1989, pp. 103-124.

[PP] F. Pop and H. Pop, An extension of the Noether-Skolem theorem, J. Pure Appl. Algebra 35 (1985), 321-328.

Department of Mathematics, DRB 155, University of Southern California, Los ANGeles, CALIFornia 90089-1113

E-mail address: vonessen@math.usc.edu 